\title{
Count per Second
}

National Cancer Institute

\section{Source}

National Cancer Institute. Count per Second. NCI Thesaurus. Code C94877.

A unit of recurring events expressed as a number of events per second. 